\title{
Modeling and simulation of air pollutant distribution in street canyon area with Skytrain stations
}

\author{
Suranath Chomcheon ${ }^{1,2}$, Nathnarong Khajohnsaksumeth ${ }^{1,2^{*}}$ (D) Benchawan Wiwatanapataphee ${ }^{3}$ and \\ Xiangyu Ge $\mathrm{Ge}^{4}$
}

"Correspondence:
nathnarong.kha@mahidol.ac.th
${ }^{1}$ Department of Mathematics,
Mahidol University, Bangkok,
Thailand
${ }^{2}$ Centre of Excellence in
Mathematics, Commission on
Higher Education (CHE), Bangkok,
Thailand
Full list of author information is
available at the end of the article

\begin{abstract}
This paper focuses on effects of the wind flow velocity on the air flow and the air pollution dispersion in a street canyon with Skytrain. The governing equations of air pollutants and air flow in this study area are the convection-diffusion equations of species concentration and the Reynolds-averaged Navier-Stokes (RANS) equations of compressible turbulent flow, respectively. Finite element method is utilized for the solution of the problem. To investigate the impact of the air flow on the pattern of air pollution dispersion, three speeds of inlet wind in three different blowing directions are chosen. The results illustrate that our model can depict the airflows and dispersion patterns for different wind conditions.
\end{abstract}

Keywords: Air pollution; Street canyon; Skytrain station

\section{Introduction}

There are many forms of pollution, including air, water, thermal, noise, soil contamination, etc. $[1,2]$. The air pollution is considered to be the world's top environmental cause of mortality [3]. It has been reported that pollution has killed many million people in the world [4]. The critical air pollutants, including carbon dioxide, lead, nitrogen dioxide, ozone particles, and sulfur dioxide [5-10], come from factories, industries, and transportation. The transportation sector is responsible for a large portion of air pollution in large urban centers due to vehicle emissions. To protect public health, understanding how to keep the air quality acceptable in street canyons or other urban areas is important. Over the last few decades, many researchers have focused on the air quality control using both experimental and numerical models. As experimental studies cannot illustrate the pattern of pollutant dispersion accurately, numerical models have thus been used as a main tool over the last few decades to investigate the local sources of air pollution in street canyon environment under several aspects such as the presence of vegetation $[11,12]$ and dust [13], the surrounding airflow, and air pollutant dispersion [14-24]. Chan et al. proposed a twodimensional model of air pollutant dispersion in an isolated street canyon [16]. Chang et al. applied the wind tunnel model to predict the pollutant dispersion in urban street canyons with different height arrangement [17]. Kim et al. studied the thermal effects on the flow and pollutant dispersion in urban street canyons [20]. In 2011, Liu proposed two

(c) The Author(s) 2019. This article is distributed under the terms of the Creative Commons Attribution 4.0 International License (http://creativecommons.org/licenses/by/4.0/), which permits unrestricted use, distribution, and reproduction in any medium, provided you give appropriate credit to the original author(s) and the source, provide a link to the Creative Commons license, and indicate if changes were made. 
numerical models to predict vehicle exhaust dispersion in urban areas with or without a wind field [25]. In his model, the effect of building and street canyon configuration and the turbulent energy produced by moving vehicles on the pollutant propagation were investigated. A few years later in 2014, numerical investigations on pollutant dispersion in street canyons with emission sources located near the ground level were performed by Madalozzo et al. using the pseudo-compressibility hypothesis of mass conservation, the Navier-Stokes equations, energy equation, and pollutant transport equation [26]. Their results show that temperature and street-canyon geometry affect both the wind flow patterns and pollutant concentration. Recently, Aristodemou et al. studied the effect of tall buildings on turbulent airflows and pollution dispersion at a seven-building site configuration using the mesh-adaptive large eddy simulation of incompressible turbulent flow [14]. Suebyat and Pochai investigated numerically air pollution in a heavy traffic area under the Skytrain platform in Bangkok, Thailand [27]. Pothiphan et al. studied the impact of wind speeds on heat transfer in a street canyon with a Skytrain station [28]. As the results obtained from existing models are mostly based on incompressible fluid flow with or without the pseudo-compressibility assumption in street canyons with rows of buildings, our understanding of traffic-related air pollution in the street canyon with Skytrain is very limited.

In this study, we investigate the effect of wind velocity on dispersion pattern of the trafficrelated air pollution in the street canyon with Skytrain. For a single-pollutant approach to air pollution, namely, carbon dioxide, governing equations of the problem in adiabatic process are the Reynolds-averaged Navier-Stokes (RANS) equations of compressible turbulent flow and the convection-diffusion equations of the $\mathrm{CO}_{2}$ concentration. Finite element method is utilized for the solution of the problem. Nine wind conditions are chosen to demonstrate the impact of airflow on the pattern of $\mathrm{CO}_{2}$ dispersion in the study region.

\section{Mathematical model}

To describe the transport of $\mathrm{CO}_{2}$ in a street canyon with Skytrain, a transport model for an adiabatic system is developed. The compressible turbulent airflow and propagation of $\mathrm{CO}_{2}$ pollutant in the street canton with Skytrain $\Omega$ region are governed by the following initial boundary value problem (IBVP) which includes the Reynolds-averaged Navier-Stokes (RANS) equations and the convection-diffusion equations of the $\mathrm{CO}_{2}$ concentration [29], namely:

- Mass conservation equation of compressible fluid:

$$
\frac{\partial \rho}{\partial t}+\nabla \cdot(\rho \vec{u})=0
$$

- Reynolds-averaged Navier-Stokes (RANS) equations:

$$
\begin{aligned}
& \rho \frac{\partial \vec{u}}{\partial t}+\rho(\vec{u} \cdot \nabla) \vec{u}= \nabla \cdot\left[-p \mathbf{I}+\mu_{e}\left(\nabla \vec{u}+(\nabla \vec{u})^{T}\right)\right. \\
&\left.-\frac{2}{3} \mu_{e}(\nabla \cdot \vec{u}) \mathbf{I}-\frac{2}{3} \rho k \mathbf{I}\right]+F, \\
& \rho \frac{\partial k}{\partial t}+\rho(\vec{u} \cdot \nabla) k=\nabla \cdot\left[\left(\mu+\frac{\mu_{T}}{\sigma_{k}}\right)\right] \nabla k+P_{k}-\rho \epsilon, \\
& \rho \frac{\partial \varepsilon}{\partial t}+\rho(\vec{u} \cdot \nabla) \varepsilon=\nabla \cdot\left[\left(\mu+\frac{\mu_{T}}{\sigma_{\varepsilon}}\right)\right] \nabla \varepsilon+C_{\varepsilon_{1}} \frac{\varepsilon}{k} P_{k}-C_{\varepsilon_{2}} \rho \frac{\varepsilon^{2}}{k},
\end{aligned}
$$


Table 1 Unknown variables

\begin{tabular}{ll}
\hline Notation & Explanation and unit \\
\hline$\rho$ & density $\left(\mathrm{kg} / \mathrm{m}^{3}\right)$ \\
$\vec{u}$ & air velocity $(\mathrm{m} / \mathrm{s})$ \\
$k$ & turbulent kinetic energy $\left(\mathrm{m}^{2} / \mathrm{s}^{2}\right)$ \\
$\varepsilon$ & turbulent dissipation rate $\left(\mathrm{m}^{2} / \mathrm{s}^{3}\right)$ \\
$c$ & concentration of carbon dioxide $\left(\mathrm{mol} / \mathrm{m}^{3}\right)$ \\
\hline
\end{tabular}

Table 2 Model constants

\begin{tabular}{lll}
\hline Constant & Value & Unit \\
\hline$C_{\varepsilon 1}$ & 1.44 & \\
$C_{\varepsilon 2}$ & 1.92 & \\
$C_{\mu}$ & 0.09 & \\
$\sigma_{k}$ & 1.00 & \\
$\sigma_{\varepsilon}$ & 1.30 & \\
$\kappa_{V}$ & 0.41 & \\
$\delta_{W}$ & 0.20 & \\
$\kappa$ & 1.40 & $(\mathrm{~Pa} \cdot \mathrm{s})$ \\
$\mu$ & $1.509716 \times 10^{-5}$ & $\left(\mathrm{~m}^{2} / \mathrm{s}\right)$ \\
$\alpha$ & 0.1592 & $(\mathrm{~m} / \mathrm{s})$ \\
$u_{\text {ref }}$ & 1.00 &
\end{tabular}

- Pollutant transport equation:

$$
\frac{\partial c}{\partial t}-\nabla \cdot(\alpha \nabla c)+\vec{u} \cdot \nabla c=S
$$

where $\rho, \vec{u}, k, \varepsilon$ and $c$ are unknown variables (see the details in Table 1 ), $\alpha$ denotes diffusion coefficient of $\mathrm{CO}_{2}$, and $F$ and $S$ respectively denote body force and pollution source, and $\mu_{e}$ and $P_{k}$ are the effective viscosity and turbulent kinetic energy production, which are respectively defined by

$$
\begin{aligned}
& \mu_{e}=\mu+\mu_{t} \quad \text { with } \mu_{t}=\rho C_{\mu} k^{2} / \varepsilon, \quad \text { and } \\
& P_{k}=\mu_{t}\left(\nabla \vec{u}:\left(\nabla \vec{u}+(\nabla \vec{u})^{T}\right)\right) .
\end{aligned}
$$

Other constant model parameters are shown in Table 2.

In equation (2), $F$ corresponding to body force due to concentration effect is defined by

$$
F=\rho \beta\left(c-c_{0}\right) \vec{g}
$$

where $\beta$ is the coefficient of volumetric expansion due to concentration variation, $\vec{g}$ is the gravity acceleration vector, and $c_{0}$ is a reference value for concentration. In adiabatic flow, pressure term $p$, a function of density $\rho$ and the ratio of specific heat $\kappa$ [30], is defined by

$$
p=\frac{1}{\kappa} \rho^{\kappa}
$$

To completely define the initial boundary value problem (IVBP), the set of initial and boundary conditions [31] is established as follows:

- The values of $\mathrm{CO}_{2}$ concentration at initial state $t=0 \mathrm{~s}$ is assumed to be zero, i.e.,

$$
c(\vec{x}, 0)=0, \quad \forall \vec{x} \in \Omega
$$


- The pollutant emission sources are from traffic vehicles located near the ground level. On the inflow boundary of $\mathrm{CO}_{2}$ pollutant, we set

$$
c(\vec{x}, t)=c_{0}, \quad \forall \vec{x} \in \partial \Omega_{S} .
$$

- On the inflow boundary of turbulent airflow $\partial \Omega_{\text {in }}$, we set

$$
\vec{u}(\vec{x}, t)=u_{0} \hat{u}, \quad k(\vec{x}, t)=\frac{3}{2}\left(u_{\mathrm{ref}} I_{T}\right)^{2}, \quad \varepsilon(\vec{x}, t)=c_{\mu} \frac{\frac{3}{4}}{L_{T}},
$$

where $\hat{u}$ is the unit vector representing wind direction, $I_{T}$ and $L_{T}$ denote turbulent length scale and turbulent intensity, respectively, defined by

$$
I_{T}=0.16 R e^{\frac{1}{8}}, \quad L_{T}=c_{\mu} \frac{k^{\frac{2}{3}}}{\varepsilon} .
$$

- On open (outlet) boundary $\partial \Omega_{\text {out }}$, the conditions are set to be

$$
\begin{aligned}
& {\left[-\frac{\rho^{\kappa}}{\kappa} \mathbf{I}+\mu_{e}\left(\nabla \vec{u}+(\nabla \vec{u})^{T}\right)-\frac{2}{3} \mu_{e}(\nabla \cdot \vec{u}) \mathbf{I}-\frac{2}{3} \rho k \mathbf{I}\right] \vec{n}=f_{0} \vec{n},} \\
& \nabla k \cdot \vec{n}=0, \quad \text { and } \quad \nabla \varepsilon \cdot \vec{n}=0 \quad \text { if } \vec{u} \cdot \vec{n} \geq 0, \\
& k(\vec{x}, t)=\frac{3}{2}\left(U_{\text {ref }} I_{T}\right)^{2} \quad \text { and } \quad \varepsilon(\vec{x}, t)=C_{\mu}^{3 / 4} \frac{k^{3 / 2}}{L_{T}} \quad \text { if } \vec{u} \cdot \vec{n}<0, \\
& -\vec{n} \cdot \alpha \nabla c=0 \quad \text { if } \vec{n} \cdot \vec{u} \geq 0, \\
& c(\vec{x}, t)=c_{0} \quad \text { if } \vec{n} \cdot \vec{u}<0 .
\end{aligned}
$$

- In addition, we apply the near-wall condition on other boundaries $\partial \Omega_{w}$, including the walls of the Skytrain and surrounding buildings, and the surface of the road and the sidewalks, i.e.,

$$
\begin{aligned}
& \vec{u} \cdot \vec{n}=0, \quad \nabla k \cdot \vec{n}=0, \quad \varepsilon=\rho \frac{C_{\mu} k^{2}}{\kappa_{v} \delta_{w}^{+} \mu}, \\
& {\left[\mu_{e}\left(\nabla \vec{u}+(\nabla \vec{u})^{T}\right)-\frac{2}{3} \mu_{e}(\nabla \cdot \vec{u}) \mathbf{I}-\frac{2}{3} \rho k \mathbf{I}\right] \vec{n}=-\rho \frac{u_{\tau}}{\delta_{w}^{+}} \vec{u}_{t},}
\end{aligned}
$$

for $\vec{u}_{t}=\vec{u}-(\vec{u} \cdot \vec{n}) \vec{n}$ and $u_{\tau}$ being the friction velocity.

\section{Finite element formulation}

The variational statement corresponding to the above IBVP is

Find $\vec{u} \in\left[H^{1}(\Omega)\right]^{3}, \rho, k, \varepsilon, c \in H^{1}(\Omega)$ that satisfy all boundary conditions, $\forall \omega_{u}, \omega_{\rho}, \omega_{k}$, $\omega_{\varepsilon}, \omega_{c} \in H_{0}^{1}(\Omega)$,

$$
\begin{aligned}
& \left(\frac{\partial \rho}{\partial t}, \omega_{\rho}\right)+\left(\nabla \cdot(\rho \vec{u}), \omega_{\rho}\right)=0, \\
& \left(\rho \frac{\partial \vec{u}}{\partial t}, \omega_{u}\right)+\left(\rho(\vec{u} \cdot \nabla) \vec{u}, \omega_{u}\right)+\left(\mu_{e} \nabla \vec{u}, \nabla \omega_{u}\right)-\left\langle\mu_{e} \frac{\partial \vec{u}}{\partial \vec{n}}, \omega_{u}\right\rangle
\end{aligned}
$$




$$
\begin{aligned}
& =-\left(\nabla \frac{\rho^{\kappa}}{\kappa} \vec{I}, \omega_{u}\right)+\left(\vec{F}, \omega_{u}\right), \\
& \left(\rho \frac{\partial k}{\partial t}, \omega_{k}\right)+\left(\rho(\vec{u} \cdot \nabla) k, \omega_{k}\right)+\left(\left(\mu+\frac{\mu_{T}}{\sigma_{k}}\right) \nabla k, \nabla \omega_{k}\right) \\
& \quad=\left(P_{k}, \omega_{k}\right)-\left(\rho \varepsilon, \omega_{k}\right), \\
& \left(\rho \frac{\partial \varepsilon}{\partial t}, \omega_{\varepsilon}\right)+\left(\rho(\vec{u} \cdot \nabla) \varepsilon, \omega_{\varepsilon}\right)+\left(\left(\mu+\frac{\mu_{T}}{\sigma_{\varepsilon}}\right) \nabla \varepsilon, \nabla \omega_{\varepsilon}\right) \\
& \quad=\left(C_{\varepsilon_{1}} \frac{\varepsilon_{1}}{k} P_{k}-C_{\varepsilon_{2}} \rho \frac{\varepsilon^{2}}{k}, \omega_{\varepsilon}\right), \\
& \left(\rho \frac{\partial c}{\partial t}, \omega_{c}\right)+\left(\alpha \nabla c, \nabla \omega_{c}\right)+\left(\vec{u} \cdot \nabla c, \omega_{c}\right)=\left(s, \omega_{c}\right),
\end{aligned}
$$

where $(u, v)=\int_{\Omega} u v d \Omega, H^{1}(\Omega)$ is the Sobolev space $W^{1,2}(\Omega)$ with norm $\|\cdot\|_{1,2, \Omega}, H_{0}^{1}(\Omega)=$ $\left\{v \mid v \in H^{1}(\Omega)\right.$ and $v=0$ on $\left.\partial \Omega\right\}$, and $L^{2}(\Omega)$ is the space of integrable functions.

The fourth term on the LHS of equation (15) is

$$
\begin{aligned}
\left\langle\mu_{e} \frac{\partial \vec{u}}{\partial \vec{n}}, \omega_{u}\right\rangle= & \int_{\partial \Omega_{\text {out }}} \omega_{u}\left(f_{0}+\left(\frac{\rho^{\kappa}}{\kappa}+\frac{2}{3} \mu_{e} \nabla \cdot \vec{u}+\frac{2}{3} \rho k\right) \mathbf{I}\right) \vec{n} d s \\
& +\int_{\partial \Omega_{w}} \omega_{u}\left(-\rho \frac{u_{\tau}}{\delta_{w}^{+}} \vec{u}_{t}+\left(\frac{2}{3} \mu_{e} \nabla \cdot \vec{u}+\frac{2}{3} \rho k\right) \mathbf{I}\right) \vec{n} d s .
\end{aligned}
$$

Applying the Galerkin approximation, we choose an $N$-dimensional subspace $H_{N} \subset$ $H^{1}(\Omega)$ for $\vec{u}, \rho, k, \varepsilon$ and $H_{0} \subset H_{0}^{1}(\Omega)$ for $\omega_{u}, \omega_{\rho}, \omega_{k}, \omega_{\varepsilon}, \omega_{c}$. Let $\left\{\phi_{j}\right\}_{j=1}^{N}$ be a set of basis functions of $H_{N}$ and $H_{0}$. Then any unknown function $f$ and test function $h$ can be expressed in the following form:

$$
f \simeq f_{N}=\sum_{j=1}^{N} f_{j}(t) \phi_{j}(\vec{x}), \quad h \simeq h_{N}=\sum_{i=1}^{N} h_{i} \phi_{i}(\vec{x}) .
$$

Here, we consider a two-dimensional problem. For a 2D linear triangular element, we have a system of nonlinear ordinary differential equations, for $i=1,2,3$,

$$
\begin{aligned}
& \sum_{j=1}^{3}\left(\int_{\Omega} \phi_{i} \phi_{j} d \Omega\right) \dot{\rho}_{j}+\left(\int_{\Omega}(\nabla \cdot \vec{u}) \phi_{i} \phi_{j} d \Omega\right) \rho_{j}=0 \\
& \sum_{j=1}^{3}\left(\int_{\Omega} \phi_{i} \phi_{j} d \Omega\right) \dot{\vec{u}}_{j}+\left(\int_{\Omega} \phi_{i}\left(\vec{u} \cdot \nabla \phi_{j}\right)+v_{e} \nabla \phi_{i} \nabla \phi_{j} d \Omega\right. \\
&\left.\quad-\frac{2}{3 \rho} \int_{\partial \Omega_{\text {out }}} \mu_{e} \phi_{i} \frac{\partial \phi_{j}}{\partial n} d s-\frac{2}{3 \rho} \int_{\partial \Omega_{w}} \mu_{e} \phi_{i} \frac{\partial \phi_{j}}{\partial n} d s\right) \vec{u}_{t} \\
&=\frac{1}{\rho} \int_{\partial \Omega_{\text {out }}} \phi_{i}\left(f_{0}+\frac{\rho^{\kappa}}{\kappa}+\frac{2}{3} \rho^{\kappa}\right) \mathbf{I} d s+\frac{1}{\rho} \int_{\partial \Omega_{w}} \phi_{i}\left(-\rho \frac{u_{\tau}}{\delta_{w}^{+}} \vec{u}_{t}+\frac{2}{3} \rho k\right) \mathbf{I} d s \\
& \quad-\frac{1}{\rho} \int_{\Omega} \phi_{i}\left(\nabla \frac{\rho^{\kappa}}{\kappa} \mathbf{I}+\vec{F}\right) d \Omega, \\
& \sum_{j=1}^{3}\left(\int_{\Omega} \phi_{i} \phi_{j} d \Omega\right) \dot{k}_{j}+\left(\int_{\Omega} \phi_{i}(\vec{u} \cdot \nabla) \phi_{j} d \Omega+\int_{\Omega}\left(v+\frac{v_{T}}{\sigma_{k}}\right) \nabla \phi_{i} \nabla \phi_{j} d \Omega\right) k_{j}
\end{aligned}
$$




$$
\begin{aligned}
& \quad=\frac{1}{\rho} \int_{\Omega} P_{k} \phi_{i} d \Omega-\int_{\Omega} \varepsilon \phi_{i} d \Omega \\
& \sum_{j=1}^{3}\left(\int_{\Omega} \phi_{i} \phi_{j} d \Omega\right) \dot{\varepsilon}_{j}+\left(\int_{\Omega} \phi_{i}(\vec{u} \cdot \nabla) \phi_{j} d \Omega+\int_{\Omega}\left(v+\frac{v_{T}}{\sigma_{\varepsilon}}\right) \nabla \phi_{i} \nabla \phi_{j} d \Omega\right) \varepsilon_{j} \\
& \quad=\int_{\Omega} \phi_{i}\left(C_{\varepsilon_{1}} \frac{\varepsilon}{\rho k} P_{k}-C_{\varepsilon_{2}} \frac{\varepsilon^{2}}{k}\right) d \Omega \\
& \sum_{j=1}^{3}\left(\int_{\Omega} \phi_{i} \phi_{j} d \Omega\right) \dot{c}_{j}+\left(\int_{\Omega} \alpha \nabla \phi_{i} \nabla \phi_{j} d \Omega+\int_{\Omega} \phi_{i} \vec{u} \cdot \nabla \phi_{j} d \Omega\right) c_{j}=\int_{\Omega} s \phi_{i} d \Omega
\end{aligned}
$$

or in matrix form as

$$
\mathbf{M}^{(e)} \dot{\mathbf{u}}+\mathbf{A}^{(e)} \mathbf{u}=\mathbf{b}^{(e)}
$$

where $\dot{\mathbf{u}}^{T}=(\dot{\rho}, \dot{\vec{u}}, \dot{k}, \dot{\varepsilon}, \dot{c})^{T}, \mathbf{u}^{T}=(\rho, \vec{u}, k, \varepsilon, c)^{T}, \mathbf{M}^{(e)}$ and $\mathbf{A}^{(e)}$ are the element matrices, and $\mathbf{b}^{(e)}$ denotes the load vector defined as follows:

$$
\begin{aligned}
& \mathbf{M}^{(e)}=\left[\begin{array}{cccccc}
M & 0 & \ldots & \ldots & \cdots & 0 \\
0 & M & 0 & & & \vdots \\
\vdots & \ddots & \ddots & \ddots & & \vdots \\
\vdots & & \ddots & \ddots & \ddots & \vdots \\
\vdots & & & 0 & M & 0 \\
0 & \cdots & \ldots & \ldots & 0 & M
\end{array}\right]_{21 \times 21} \\
& M=\left(m_{i j}\right)_{3 \times 3}=\int_{\Omega} \phi_{i} \phi_{j} d \Omega, \\
& \mathbf{A}^{(e)}=\left[\begin{array}{ccccc}
A_{\rho} & 0 & \cdots & \cdots & 0 \\
0 & A_{\vec{u}} & 0 & & \vdots \\
\vdots & 0 & A_{k} & 0 & \vdots \\
\vdots & & 0 & A_{\varepsilon} & 0 \\
0 & \cdots & \cdots & 0 & A_{c}
\end{array}\right]_{21 \times 21} \\
& A_{\rho}=\left({ }_{\rho} a_{i j}\right)_{3 \times 3}, \quad{ }_{\rho} a_{i j}=\int_{\Omega}(\nabla \cdot \vec{u}) \phi_{i} \phi_{j} d \Omega, \\
& A_{\vec{u}}=\left({ }_{\vec{u}} a_{i j}\right)_{3 \times 3}, \\
& \vec{u} a_{i j}=\int_{\Omega} \phi_{i}\left(\vec{u} \cdot \nabla \phi_{j}\right)+v_{e} \nabla \phi_{i} \nabla \phi_{j} d \Omega-\frac{2}{3 \rho} \int_{\partial \Omega_{\text {out }}} \mu_{e} \phi_{i} \frac{\partial \phi_{j}}{\partial n} d s-\frac{2}{3 \rho} \int_{\partial \Omega_{w}} \mu_{e} \phi_{i} \frac{\partial \phi_{j}}{\partial n} d s, \\
& A_{k}=\left({ }_{k} a_{i j}\right)_{3 \times 3}, \quad{ }_{k} a_{i j}=\int_{\Omega} \phi_{i}(\vec{u} \cdot \nabla) \phi_{j} d \Omega+\int_{\Omega}\left(v+\frac{\nu_{T}}{\sigma_{k}}\right) \nabla \phi_{i} \nabla \phi_{j} d \Omega, \\
& A_{\varepsilon}=\left({ }_{\varepsilon} a_{i j}\right)_{3 \times 3}, \quad{ }_{\varepsilon} a_{i j}=\int_{\Omega} \phi_{i}(\vec{u} \cdot \nabla) \phi_{j} d \Omega+\int_{\Omega}\left(v+\frac{v_{T}}{\sigma_{\varepsilon}}\right) \nabla \phi_{i} \nabla \phi_{j} d \Omega, \\
& A_{c}=\left({ }_{c} a_{i j}\right)_{3 \times 3}, \quad{ }_{c} a_{i j}=\int_{\Omega} \alpha \nabla \phi_{i} \nabla \phi_{j} d \Omega+\int_{\Omega} \phi_{i} \vec{u} \cdot \nabla \phi_{j} d \Omega
\end{aligned}
$$




$$
\begin{gathered}
\mathbf{b}^{(e)}=\left[\begin{array}{l}
b_{\rho} \\
b_{\vec{u}} \\
b_{k} \\
b_{\varepsilon} \\
b_{c}
\end{array}\right]_{21 \times 1}, \\
b_{\rho}=\left({ }_{\rho} b_{i j}\right)_{3 \times 3}, \quad{ }_{\rho} b_{i j}=0, \\
b_{\vec{u}}=\left({ }_{\vec{u}} b_{i j}\right)_{3 \times 3}, \quad{ }^{\prime}, \phi_{i j}\left(f_{0}+\frac{\rho^{\kappa}}{\kappa}+\frac{2}{3} \rho^{\kappa}\right) \mathbf{I} d s+\frac{1}{\rho} \int_{\partial \Omega_{w}} \phi_{i}\left(-\rho \frac{u_{\tau}}{\delta_{w}^{+}} \vec{u}_{t}+\frac{2}{3} \rho k\right) \mathbf{I} d s \\
\quad-\frac{1}{\rho} \int_{\Omega} \phi_{i}\left(\nabla \frac{\rho^{\kappa}}{\kappa} \mathbf{I}+\vec{F}\right) d \Omega, \\
b_{k}=\left({ }_{k} b_{i j}\right)_{3 \times 3}, \quad{ }_{k} b_{i j}=\frac{1}{\rho} \int_{\Omega} P_{k} \phi_{i} d \Omega-\int_{\Omega} \varepsilon \phi_{i} d \Omega, \\
b_{\varepsilon}=\left({ }_{\varepsilon} b_{i j}\right)_{3 \times 3}, \quad{ }_{\varepsilon} b_{i j}=\int_{\Omega} \phi_{i}\left(C_{\varepsilon_{1}} \frac{\varepsilon}{\rho k} P_{k}-C_{\varepsilon_{2}} \frac{\varepsilon^{2}}{k}\right) d \Omega, \\
b_{c}=\left({ }_{c} b_{i j}\right)_{3 \times 3}, \quad{ }_{c} b_{i j}=\int_{\Omega} s \phi_{i} d \Omega .
\end{gathered}
$$

By assembling all element matrices and all element vectors, we obtain the global system

$$
\mathbf{M} \dot{\mathbf{u}}+\mathbf{A u}=\mathbf{F},
$$

which can be solved by a time integration scheme at any instant of time:

$$
\mathbf{M} \frac{\mathbf{u}_{n+1}-\mathbf{u}_{n}}{\triangle t}+\triangle\left(\mathbf{u}_{n}\right) \mathbf{u}_{n}=\mathbf{F}_{n}
$$

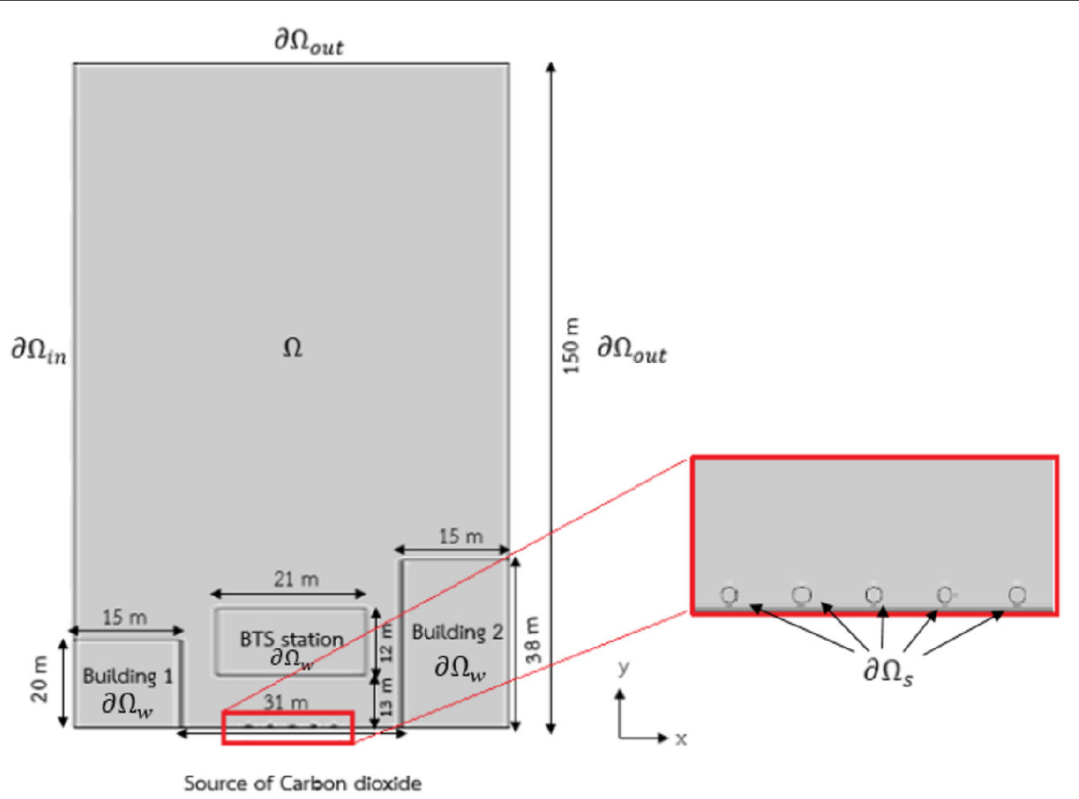

Figure 1 Computational domain 


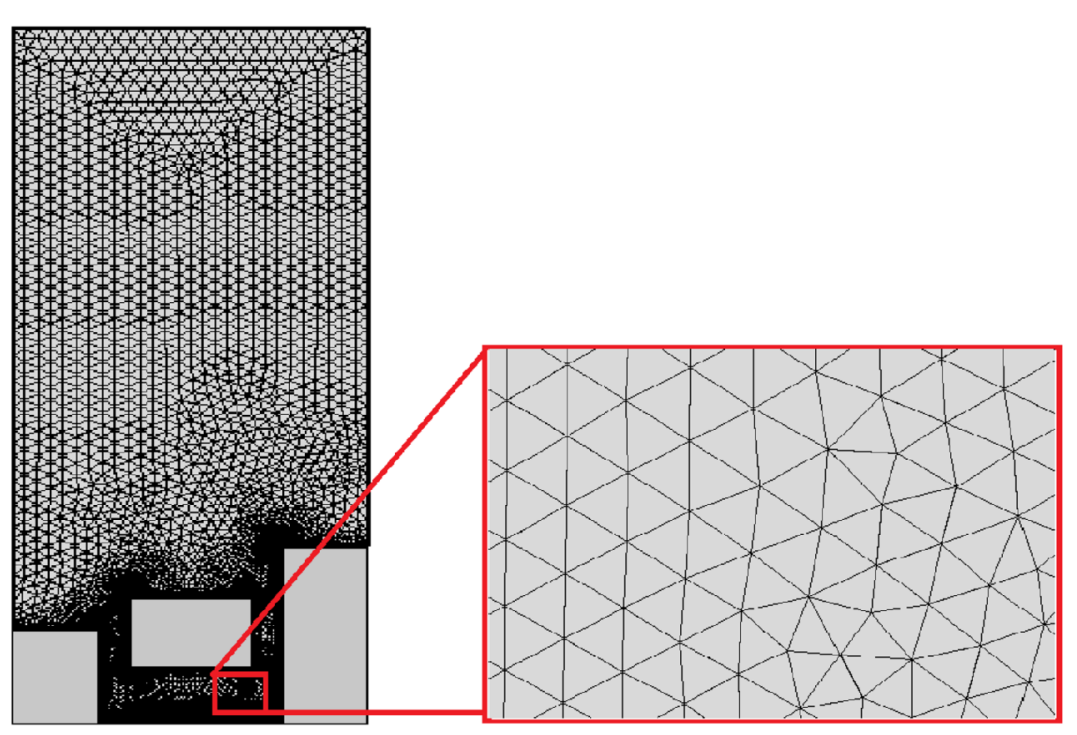

Figure 2 Domain mesh

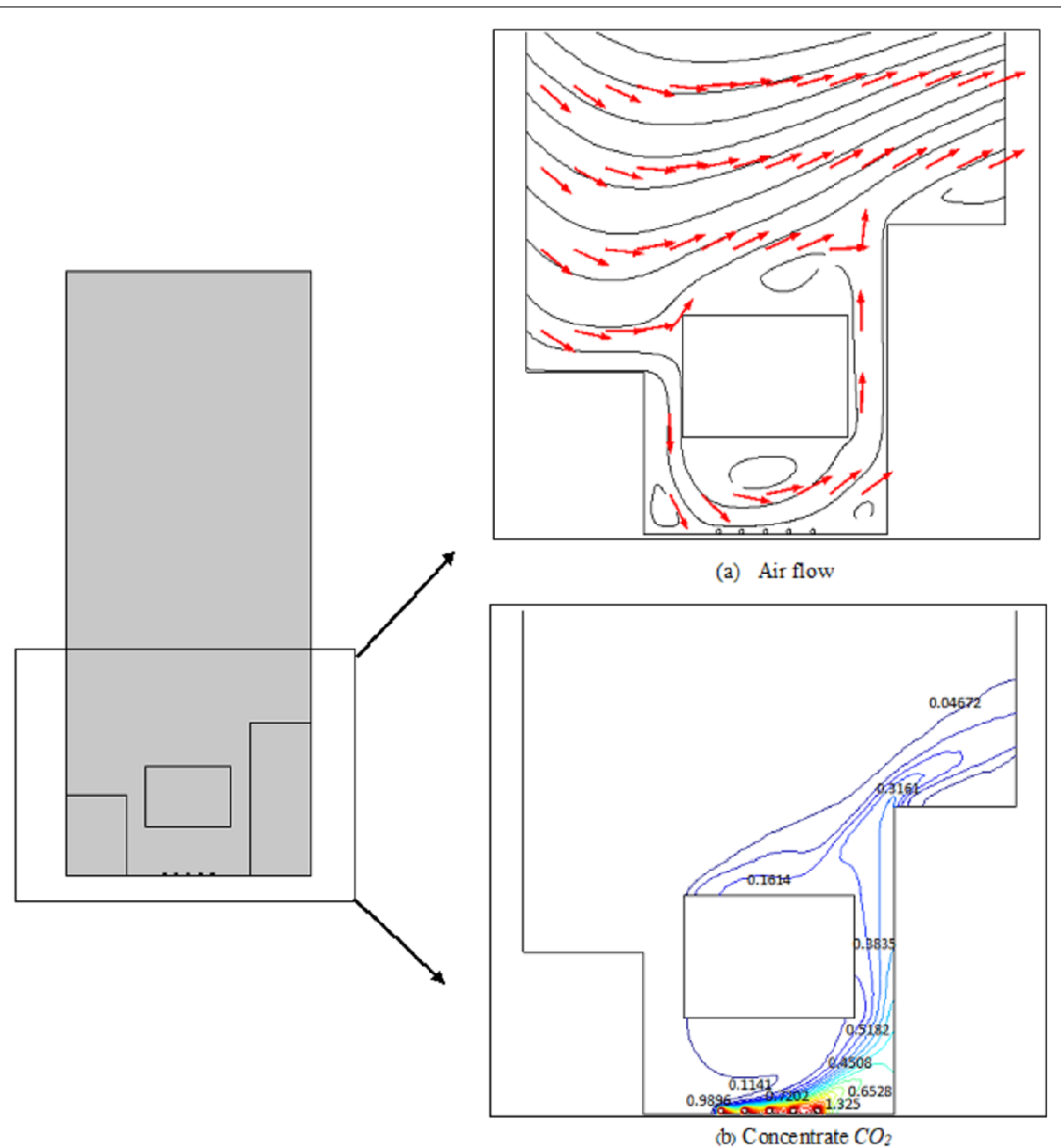

Figure 3 Velocity field and streamlines of wind and contour lines of $\mathrm{CO}_{2}$ concentration obtained from the model with the inlet wind magnitude of $5.40 \mathrm{knots}(10 \mathrm{~km} / \mathrm{h})$ and the wind blowing from the southeast at 135 degrees 


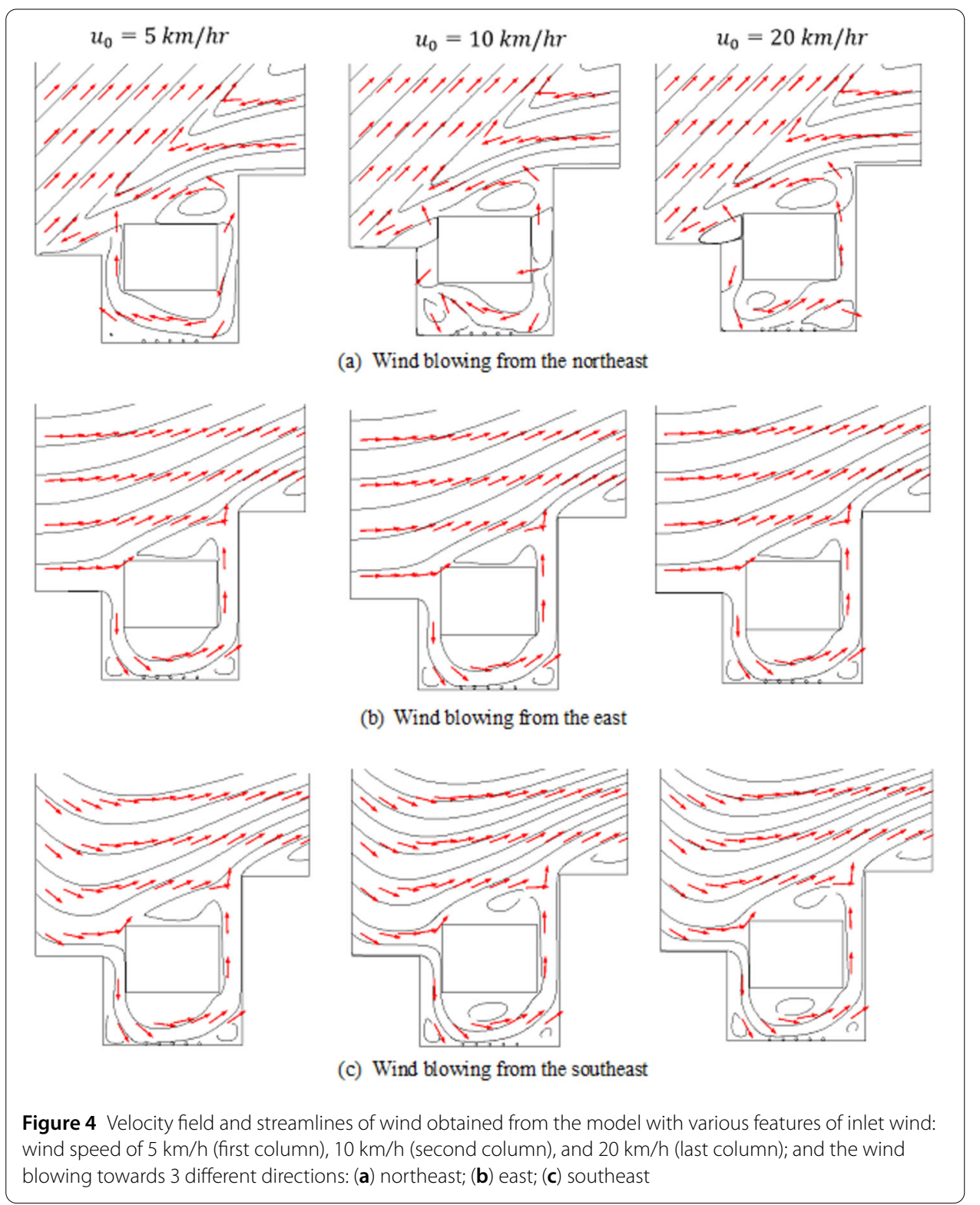

\section{Numerical result}

This section investigates numerically the effect of the airflow on air pollution dispersion within a street canyon having a Skytrain platform, 20- and 38-meter-tall buildings, and two 5-meter-width sidewalks. COMSOL Multiphysics Modelling software is applied to simulate the finite element approximation of solutions [29]. We assume that no airflow and pollutant emission occur in solid structures including buildings, a Skytrain platform and sidewalks, and emissions of carbon dioxide are only from traffic vehicles under the Skytrain. Figure 1 presents two-dimensional Skytrain-street-canyon analysis with five emission sources on the ground level. The Skytrain station with width of $21 \mathrm{~m}$ and height of $12 \mathrm{~m}$ is located $13 \mathrm{~m}$ above the traffic level. The computational domain with a nonuniform finite element mesh is shown in Fig. 2.

The emission rate of $0.068 \mathrm{~mol} / \mathrm{m}^{3}$, equivalent to the amount of pollutant from 20 vehicles, is chosen to analyze the impact of wind velocity on concentration distribution of air 

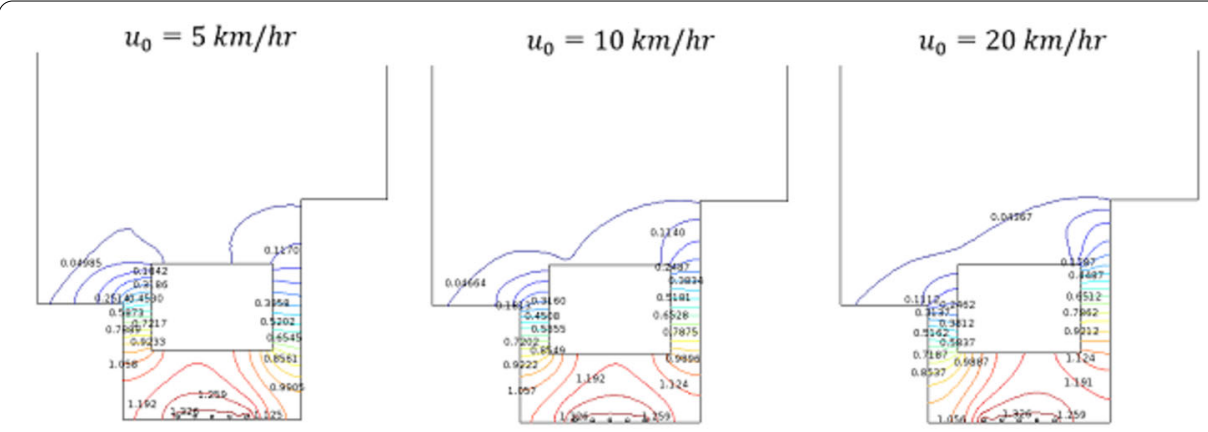

(a) Wind blowing from the northeast
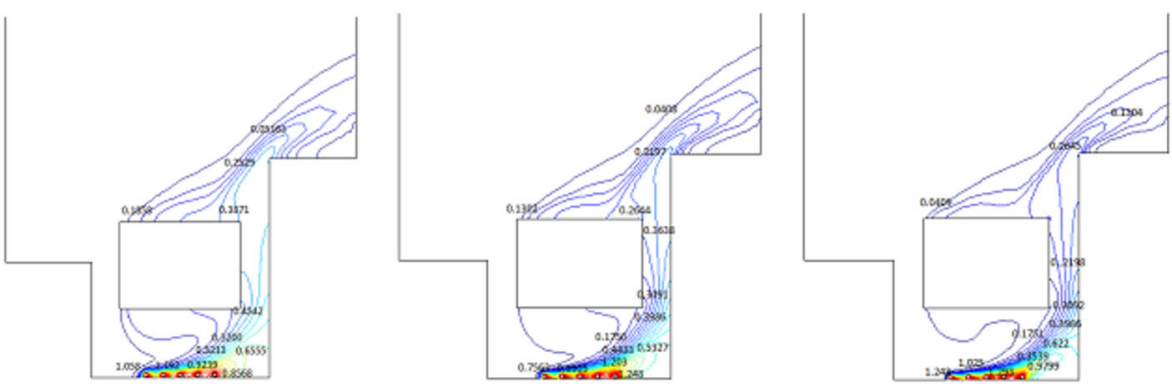

(b) Wind blowing from the east
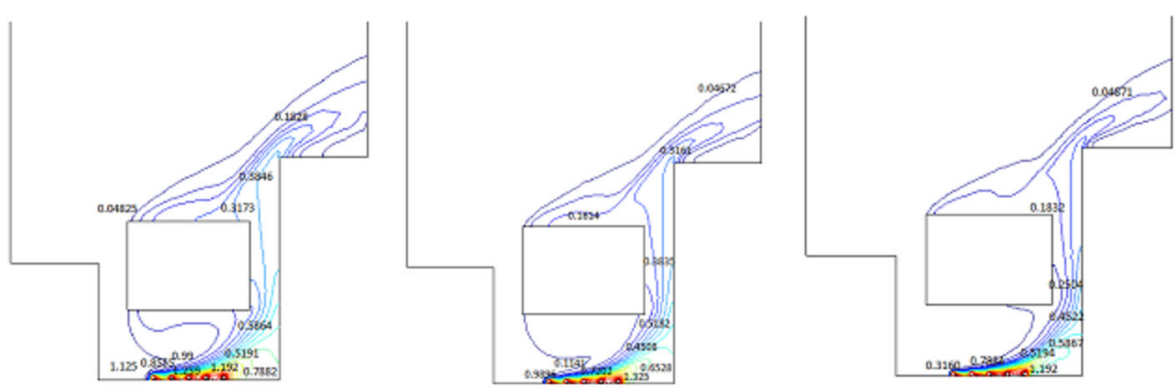

(c) Wind blowing from the southeast

Figure 5 Contour lines of $\mathrm{CO}_{2}$ concentration obtained from the model with various features of inlet wind: wind speed of $5 \mathrm{~km} / \mathrm{h}$ (first column), $10 \mathrm{~km} / \mathrm{h}$ (second column), and $20 \mathrm{~km} / \mathrm{h}$ (last column); and wind blowing towards 3 different directions: (a) northeast; (b) east; (c) southeast

pollutants. Air is assumed to flow into the study region from the left-side rooftop. Figure 3 shows wind velocity with its streamlines and contour lines of carbon dioxide concentration obtained from the model with inflow wind speed of $10 \mathrm{~km} / \mathrm{h}$ from southeast. The wind splits into two parts, upstream and downstream, when it approaches the Skytrain station. The downstream part flows into the Skytrain cavity in which three wind-circulation zones occur. A large circulation zone is below the Skytrain station and two small circulation zones are next to the buildings. This wind flow field has a significant impact on the dispersion and distribution of air pollutants in the Skytrain region. Air pollutants from traffic vehicles propagate into three wind-circulation zones and the area next to the building and Skytrain station. Figures 4 and 5 illustrate the effect of inflow features on the airflow pattern and distribution of air pollutant concentration, respectively. The inflow wind with speed of 5,10 , and $20 \mathrm{~km} / \mathrm{h}$ gives different patterns of airflow and air pollutant concentration compared to those obtained from the models with other inflow conditions. The 

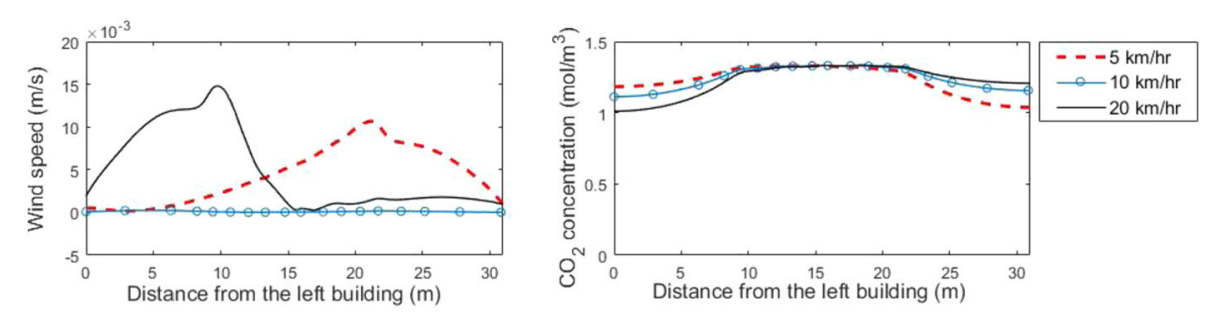

(a) Wind blowing from the northeast
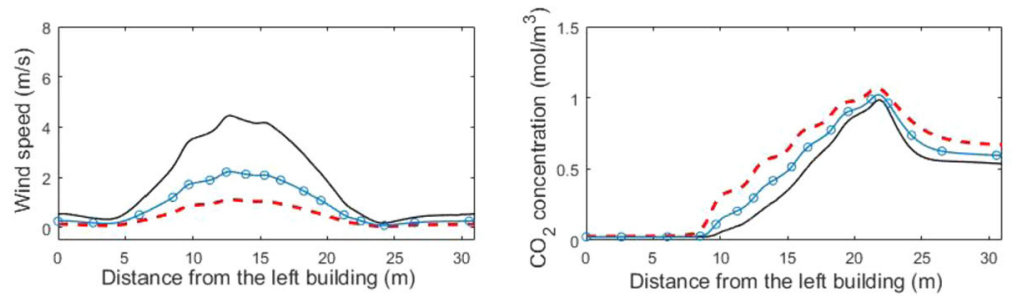

(b) Wind blowing from the east
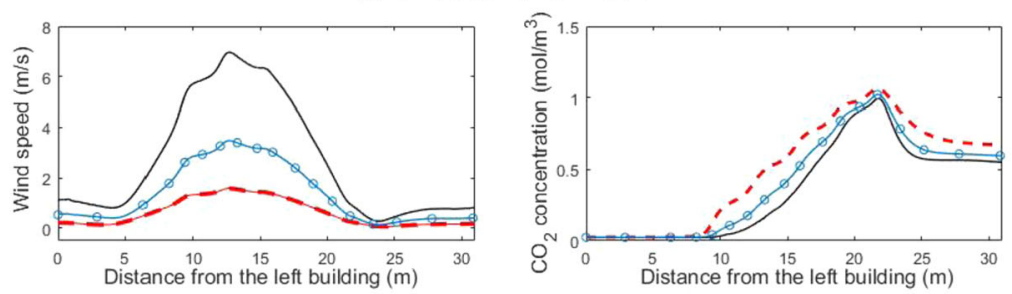

(c) Wind blowing from the southeast

Figure 6 Comparison of wind-velocity profiles and $\mathrm{CO}_{2}$-concentration profiles at 1.5-meter height above the ground obtained from model with various conditions of the inlet wind speed and its blowing direction

air pollutant concentration obtained from the model with wind blowing from northeast spreads throughout the Skytrain cavity and both sides of the Skytrain station while it distributes to the right-sided region for the inflow wind blowing from east and southeast. Figures 6 and 7 compare the profiles of wind velocity and air pollutant concentration at 1.5- and 2-meter heights above the ground obtained from the inflow wind models using 3 different speeds and 3 different wind blowing directions. The results indicate that a lower speed of the inflow wind blowing from both east and southeast gives a higher air pollutant concentration in the Skytrain cavity. It is also found that there is no significant difference of $\mathrm{CO}_{2}$ concentration in the Skytrain cavity for different speeds of the inflow wind blowing from the northeast.

\section{Conclusion}

This paper demonstrates two-dimensional analysis of air pollution in the street canyon with a Skytrain platform using numerical modeling based on an initial boundary value problem (IBVP) governed by the Reynolds-averaged Navier-Stokes (RANS) equations of compressible turbulent airflow and the convection-diffusion equations of species concentration. The results indicate that the inflow wind on the rooftop of the building affects the airflow pattern in the Skytrain street canyon, leading to a change in concentration of air pollutants. The low wind speed is responsible for the concentrations of pollutants in the area under the Skytrain platform. Our modeling technique may help civil engineers to find technical solutions which will reduce air pollution emissions to more acceptable limits. 

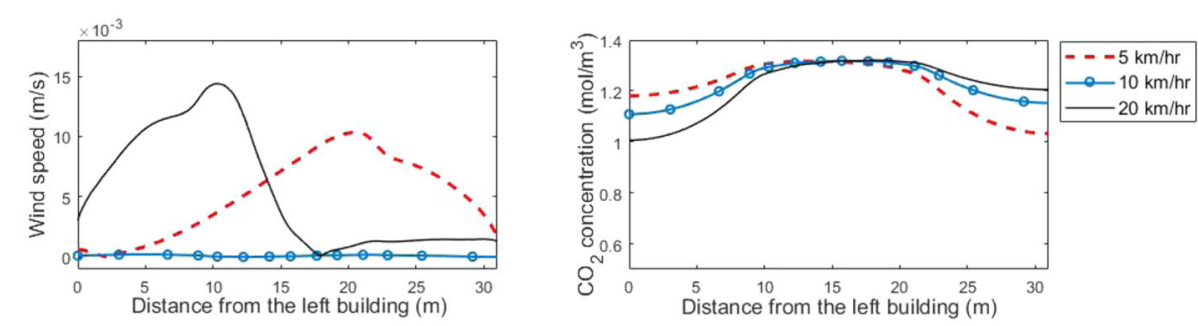

(a) Wind blowing from the northeast
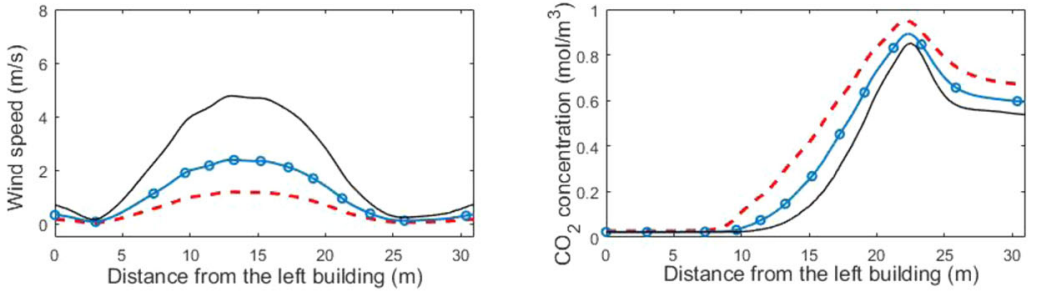

(b) Wind blowing from the east
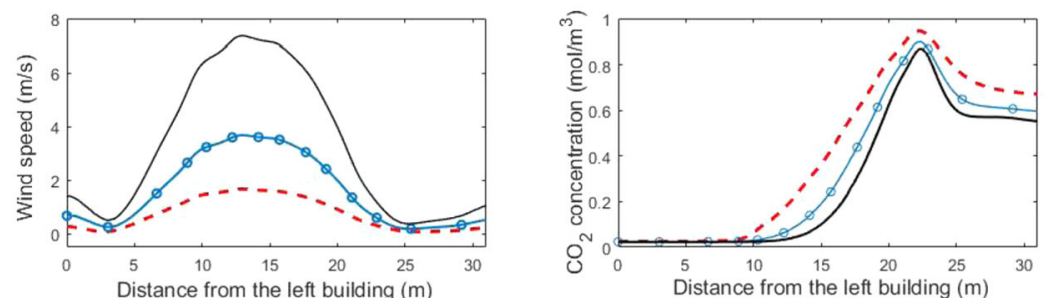

(c) Wind blowing from the southeast

Figure 7 Comparison of wind-velocity profiles and $\mathrm{CO}_{2}$-concentration profiles at 2-meter height above the ground obtained from model with various conditions of the inlet wind speed and its blowing direction

\section{Funding}

We would like to thank the Department of Mathematics, Faculty of Science, Mahidol University, and the Center of Excellence in Mathematics, for their financial support.

\section{Competing interests}

The authors declare that they have no competing interests.

\section{Authors' contributions}

The first author contributed the numerical simulation. The second and third authors formulated a mathematical model, wrote the paper and were responsible for examining results, editing and revising the manuscript. The fourth author verified the analytical methods. All authors read and approved the final manuscript.

\section{Author details}

${ }^{1}$ Department of Mathematics, Mahidol University, Bangkok, Thailand. ${ }^{2}$ Centre of Excellence in Mathematics, Commission on Higher Education (CHE), Bangkok, Thailand. ${ }^{3}$ School of Electrical Engineering, Computing and Mathematical Science, Curtin University, Perth, Australia. ${ }^{4}$ Department of Finance, Wuhan Technology and Business University, Wuhan, China.

\section{Publisher's Note}

Springer Nature remains neutral with regard to jurisdictional claims in published maps and institutional affiliations.

Received: 26 July 2019 Accepted: 16 October 2019 Published online: 30 October 2019

\section{References}

1. Brunekreef, B., Holgate, S.T.: Air pollution and health. Lancet 360(9341), 1233-1242 (2002)

2. Maynard, R.: Key airborne pollutants - the impact on health. Sci. Total Environ. 334, 9-13 (2004)

3. Global IGBP change: urban air pollution-a new look at an old problem. http://www.igbp.net/news/features/ features/urbanairpollutionanewlookatanoldproblem.5.19895cff13e9f675e253fo.html

4. Health effects. https://www.airqualitynow.eu/pollution_health_effects.php

5. Sangeetha, A., Amudha, T.: A study on estimation of $\mathrm{CO}_{2}$ emission using computational techniques. In: 2016 IEEE International Conference on Advances in Computer Applications (ICACA), pp. 244-249. IEEE, New York (2016)

6. Kongritti, N.: Power-based motor-vehicles model emission of air pollutants from Chalongrat expressway compared to road ground level by using power-based motor-vehicle model. Asia-Pac. J. Sci. Technol. 19(5), 645-655 (2014) 
7. Is $\mathrm{CO}_{2}$ a pollutant? https://skepticalscience.com/co2-pollutant-advanced.htm

8. The effects of carbon dioxide on air pollution.

https://sciencing.com/list-5921485-effects-carbon-dioxide-air-pollution.htm

9. The criteria air pollutants. https://en.wikipedia.org/wiki/Criteria_air_pollutants

10. Air pollution. https://www.nationalgeographic.com/environment/global-warming/pollution

11. Abhijith, K.V., Kumar, P., Gallagher, J., McNabola, A., Baldauf, R., Pilla, F., Broderick, B., Sabatino, S.D., Pulvirenti, B.: Air pollution abatement performances of green infrastructure in open-road and built-up street canyon environments - a review. Atmos. Environ. 162, 71-86 (2017)

12. Janhäll, S.: Review on urban vegetation and particle air pollution-deposition and dispersion. Atmos. Environ. 105, 130-137 (2015)

13. Rahman, I.A., Putra, J.C.P., Asmi, A.: Modelling of particle transmission in laminar flow using COMSOL Multiphysics. ARPN J. Eng. Appl. Sci. 11, 6630-6637 (2006)

14. Aristodemou, E., Boganegra, L.M., Mottet, L., Pavlidis, D., Constantinou, A., Pain, C., Robins, A., ApSimon, H.: How tall buildings affect turbulent air flows and dispersion of pollution within a neighbourhood. Environ. Pollut. 233, 782-796 (2018)

15. Baik, J.-J., Kim, J.-J.: A numerical study of flow and pollutant dispersion characteristics in urban street canyons. J. Appl. Meteorol. 38(11), 1576-1589 (1999)

16. Chan, T., Dong, G., Leung, C., Cheung, C., Hung, W.: Validation of a two-dimensional pollutant dispersion model in an isolated street canyon. Atmos. Environ. 36(5), 861-872 (2002)

17. Chang, C.-H., Lin, J.-S., Cheng, C.-M., Hong, Y.-S.: Numerical simulations and wind tunnel studies of pollutant dispersion in the urban street canyons with different height arrangement. J. Mar. Sci. Technol. 21(2), 119-126 (2013)

18. Huang, H., Akutsu, Y., Arai, M., Tamura, M.: A two-dimensional air quality model in an urban street canyon: evaluation and sensitivity analysis. Atmos. Environ. 34(5), 689-698 (2000)

19. Ibrahim, A., Altinişik, K., Keskin, A.: The pollutant emissions from diesel-engine vehicles and exhaust aftertreatment systems. Clean Technol. Environ. Policy 17(1), 15-27 (2015)

20. Kim, J.-J., Baik, J.-J.: A numerical study of thermal effects on flow and pollutant dispersion in urban street canyons. J. Appl. Meteorol. 38(9), 1249-1261 (1999)

21. Li, L., Yang, L., Zhang, L.-J., Jiang, Y.: Numerical study on the impact of ground heating and ambient wind speed on flow fields in street canyons. Adv. Atmos. Sci. 29(6), 1227-1237 (2012)

22. Mei, S.-J., Liu, C.-W., Liu, D., Zhao, F.-Y., Wang, H.-Q., Li, X.-H.: Fluid mechanical dispersion of airborne pollutants inside urban street canyons subjecting to multi-component ventilation and unstable thermal stratifications. Sci. Total Environ. 565, 1102-1115 (2016)

23. Miao, Y., Liu, S., Zheng, Y., Wang, S., Li, Y.: Numerical study of traffic pollutant dispersion within different street canyon configurations. Adv. Meteorol. 2014, Article ID 458671 (2014)

24. Oyjinda, P., Pochai, N.: Numerical simulation to air pollution emission control near an industrial zone. Adv. Math. Phys. 2017, Article ID 5287132 (2017)

25. Liu, W.: Numerical models for vehicle exhaust dispersion in complex urban areas. Int. J. Numer. Methods Fluids 67 787-804 (2011)

26. Madalozzo, D.M.S., Braun, A.L., Awruch, A.M., Morsch, I.B.: Numerical simulation of pollutant dispersion in street canyons: geometric and thermal effects. Appl. Math. Model. 38(24), 5883-5909 (2014)

27. Suebyat, K., Pochai, N.: Numerical simulation for a three-dimensional air pollution measurement model in a heavy traffic area under the Bangkok Skytrain platform. Abstr. Appl. Anal. 2018, Article ID 9025851 (2018)

28. Pothiphan, S., Khajohnsaksumeth, N., Wiwatanapataphee, B.: Effect of the wind speeds on heat transfer in a street canyon with a Skytrain station. Adv. Differ. Equ. 2019, 258 (2019)

29. COMSOL Multiphysics V. 5, Comsol.com. COMSOL AB

30. Pořízkovà, P., Kozel, K., Horáček, J.: Numerical solution of compressible and incompressible unsteady flows in channel inspired by vocal tract. J. Comput. Appl. Math. 270, 323-329 (2014)

31. Wu, Y.H., Wiwatanapataphee, B.: Modelling of turbulent flow and multi-phase heat transfer under electromagnetic force. Discrete Contin. Dyn. Syst. 8, 695-706 (2007)

\section{Submit your manuscript to a SpringerOpen ${ }^{\circ}$ journal and benefit from:}

- Convenient online submission

- Rigorous peer review

- Open access: articles freely available online

- High visibility within the field

- Retaining the copyright to your article

Submit your next manuscript at $>$ springeropen.com 